\title{
A gene transcription signature associated with hormone independence in a subset of both breast and prostate cancers Chad J Creighton*
}

\author{
Address: Department of Medicine, Dan L. Duncan Cancer Center Division of Biostatistics, Baylor College of Medicine, Houston, TX 77030, USA \\ Email: Chad J Creighton* - creighto@bcm.tmc.edu \\ * Corresponding author
}

Published: 28 June 2007

BMC Genomics 2007, 8:199 doi:10.1186/147|-2164-8-199

This article is available from: http://www.biomedcentral.com//47I-2/64/8/199

(C) 2007 Creighton; licensee BioMed Central Ltd.

This is an Open Access article distributed under the terms of the Creative Commons Attribution License (http://creativecommons.org/licenses/by/2.0), which permits unrestricted use, distribution, and reproduction in any medium, provided the original work is properly cited.
Received: 12 March 2007

Accepted: 28 June 2007

\begin{abstract}
Background: The development of resistance to hormone therapy in both breast and prostate cancers is attributed to tens of thousands of patient deaths every year.

Results: From analyses of global gene expression profile data, a nonrandom amount of overlap was observed between the set of genes associated with estrogen receptor negative (ER-), hormone independent breast cancer and the set of genes associated with androgen independent (Al) prostate cancer. A set of $8 \mathrm{I}$ genes was identified that were differentially expressed between ERand ER+ clinical breast tumors and breast cancer cell lines and that showed concordant expression in Al versus AS (androgen sensitive) prostate cell lines. This common gene signature of hormone independence was used to identify a subset of clinically localized primary prostate tumors that shared extensive similarities in gene transcription with both ER- breast and AI prostate cell lines and that tended to show concurrent deactivation of the androgen signaling pathway. Both ERbreast and $\mathrm{Al}$ prostate cell lines were significantly enriched for transcriptional targets of signaling via epidermal growth factor receptor (EGFR).
\end{abstract}

Conclusion: This study indicates that the growth- and survival-promoting functions of hormone receptors can be bypassed in a subset of both breast and prostate cancers by the same growth factor signaling pathways, which holds implications for the use of targeted therapy regimens.

\section{Background}

In 2006, on the order of 234,000 men and 213,000 women were diagnosed with prostate cancer and breast cancer, respectively, and about 27,000 men and 41,000 women died (American Cancer Society statistics). Steroid hormone receptor signaling has been linked to all stages of prostate and breast carcinogenesis $[1,2]$. Initial treatment of clinically localized prostate cancer (PCA) and invasive breast cancer (IBC) usually involves surgical removal of the cancerous tissue or radiation therapy. The clinical use of adjuvant anti-androgen therapy in PCA and of anti-estrogen therapy in IBC has aided greatly in pro- longing or preventing disease recurrence, as the majority of these cancers, at least initially, depend upon their associated hormones for growth. However, significant fractions of PCA and IBC either initially present as hormone independent or develop hormone independence over the course of anti-hormone therapy $[3,4]$. In the case of IBC, hormone independence correlates closely with expression of the estrogen receptor alpha (ER), with $30-35 \%$ of IBC being ER-negative (ER-) and exhibiting no requirement of estrogen for growth $[3,5]$. Except for the fraction of ERIBC that express HER2/neu, no targeted therapy is currently in widespread use for ER- IBC. In the case of 
advanced PCA, androgen ablation therapy effectively results in tumor regression over the short-term; in most cases, however, the recurrence of highly aggressive and metastatic prostate cancer that is resistant to hormone therapy occurs as a result $[1,4]$.

Breast and prostate cancers share much in common with each other, in that they both manifest as either hormone dependent or independent. The hypothesis explored in this present study is that the molecular mechanisms of acquirement of hormone independence are similar between IBC and PCA [4]. Global gene expression profiling studies, carried out in breast and prostate cancers separately, indicate that on the order of hundreds or even thousands of genes might be involved in hormone independence in each disease [6-8]. If a select set of genes common to hormone independent breast and prostate cancers could be identified, it might be indicative of a core transcriptional program on which attention could be focused. The main strategy of this study was to look for patterns of enrichment, i.e. to look for a non-random amount of shared overlap between distinct sets of genes associated separately with either breast or prostate cancers. Such a pattern of enrichment may involve only a fraction of the genes from each cancer type and yet may hold biological and clinical significance.

\section{Results}

A gene expression signature of ER-, hormone-independent clinical breast tumors that is partially manifested in ERbreast cancer cell lines

The basic approach of this study was to first derive separate gene expression signature patterns of hormone independence from breast and prostate cancers and then to determine whether the two signatures shared significant similarity with each other. A gene transcription signature of ER- (hormone-independent) versus ER+ (hormonedependent) invasive breast cancer (IBC) was defined by selecting genes showing differential expression $(p<0.01)$ in each of two independent mRNA profile datasets of 295 clinical IBC (the dataset from van de Vijver et al., ref [9], with 69 profiles from ER- tumors) and 286 tumors (the dataset from Wang et al., ref [10], with 77 ER-profiles). Of the 2486 uniquely identified genes in this ER-status signature, 1332 were higher in the ER- tumors.

The expression patterns of the ER-status gene signature as derived from clinical IBC were further examined in an additional dataset of breast cancer cell lines from Bild $e t$ al. [11], which consisted of 28 mRNA profiles representing 18 different cell lines (ten of them ER-). As expected, a significant portion of the clinical ER-status signature showed the corresponding expression patterns in cell lines. Out of the 1332 genes found to be higher in ERclinical tumors, 223 were higher in ER- cell lines $(p<0.01)$, while 848 showed no such trend $(p>0.1)$. The intersection of the clinical and cell line breast cancer signatures was termed the "core breast ER-status signature" and consisted of 223 ER- genes (Venn diagram represented in Figure 1) and $194 \mathrm{ER}+$ genes $(p<0.01$ in each of Bild, van de Vijver, and Wang datasets). This core signature pattern was considered to be independent of the tissue or environmental context. The suggestion that a set of genes associated with hormone independence in breast cancer were regulated in vivo but not in vitro seemed intriguing but was not further explored in this study.

The expression patterns of the clinical breast ER-status signature were visualized as heat maps in both the clinical and cell culture profile datasets (Figure 2). From the heat map representation, it was apparent that a small fraction of the breast tumors in the Wang profile dataset that were classified as ER+ by immunohistochemistry showed gene expression patterns more characteristic of ER- tumors, as

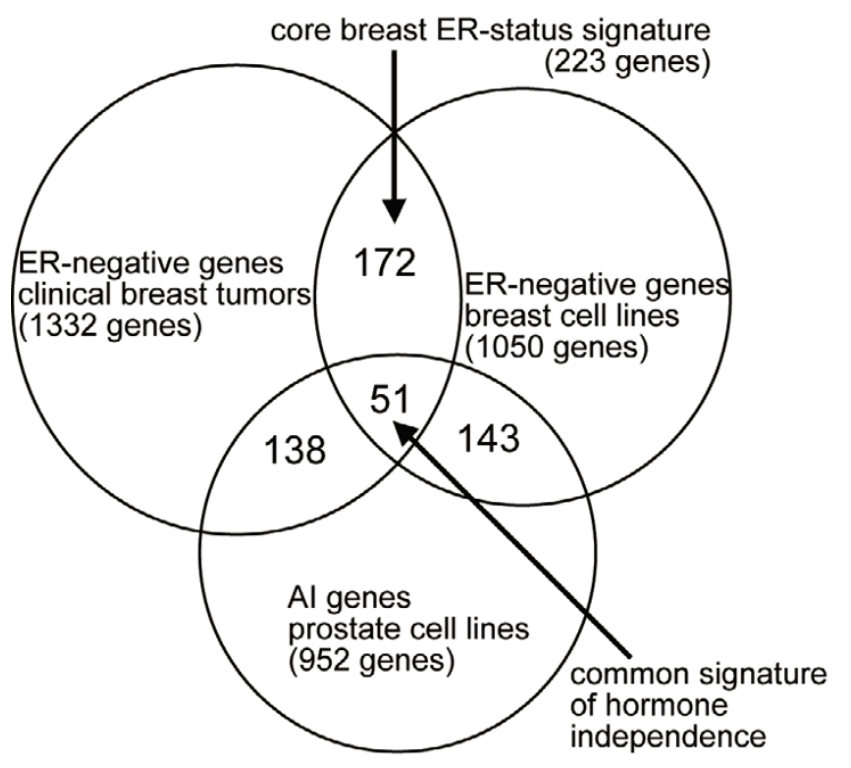

Figure I

Genes associated with hormone independence in breast cancer share significant overlap with genes associated with hormone independence in prostate cancer. Venn diagram showing the overlap between the following sets of genes: (I) genes more highly expressed in clinical ER- over ER+ breast tumors $(p<0.01$ in each of the RNA profile datasets from van de Vijver et al. and Wang et al.), (2) genes more highly expressed in ER- over ER+ cell lines $(p<0.01$ in the profile dataset from Bild et al.), and (3) genes more highly expressed in androgen independent (AI) over androgen sensitive (AS) prostate cell lines $(p<0.05$ in the dataset from Zhao et al.). Core breast ER-status signature, genes shared between the clinical breast tumor and breast cancer cell line sets. Common signature of hormone independence, genes shared between all three sets. 
well as low ER mRNA. Similarly, one particular breast cancer cell line, HCC1428, was designated as ER+ [12] but from its profile appeared more similar to ER-cell lines. In the heat map representation (Figure 2), the ER- tumor and cell line profiles were ordered by increasing similarity to the overall ER- expression pattern. The small fraction of ER- tumors which did not fit the pattern tended to have high RNA expression of the HER2 oncogene. In addition, the ER-, HER2+ SKBR3 cell line did not fit the pattern of the other ER-cell lines. All of this indicated that the core breast ER-status signature (Figure 2) was a pattern of ER-, HER2- breast cancer, with the ER-, HER2+ breast cancers having a different pattern, as has been indicated in previous expression profiling studies $[6,7,12]$.

\section{A gene expression signature common to ER- breast cancer} and Al, hormone-independent prostate cancer cell lines Unlike breast cancer, there are currently no well-defined molecular markers of hormone-independent prostate cancer. For example, expression of the androgen receptor (AR) does not appear to correlate with response to hormone therapy in prostate cancer, and AR protein is expressed fairly homogeneously in primary tumors, recurrent local tumors, and metastases [1]. It was therefore difficult to define a signature of hormone-independence from profiles of clinical prostate tumors alone. However, a number of prostate cancer cell lines have been established that are classified as either androgen sensitive (AS), which respond to androgen stimulation, and androgen insensitive (AI), which do not respond. As discussed below (see Discussion and [4]), there are several known mechanisms by which prostate cancers may develop resistance to hormone therapy. While the cell lines considered here were entirely androgen pathway independent, other prostate cancers develop hypersensitivity to androgen receptor pathway signaling; this latter type of hormone therapy resistant cancer was not considered in this study.
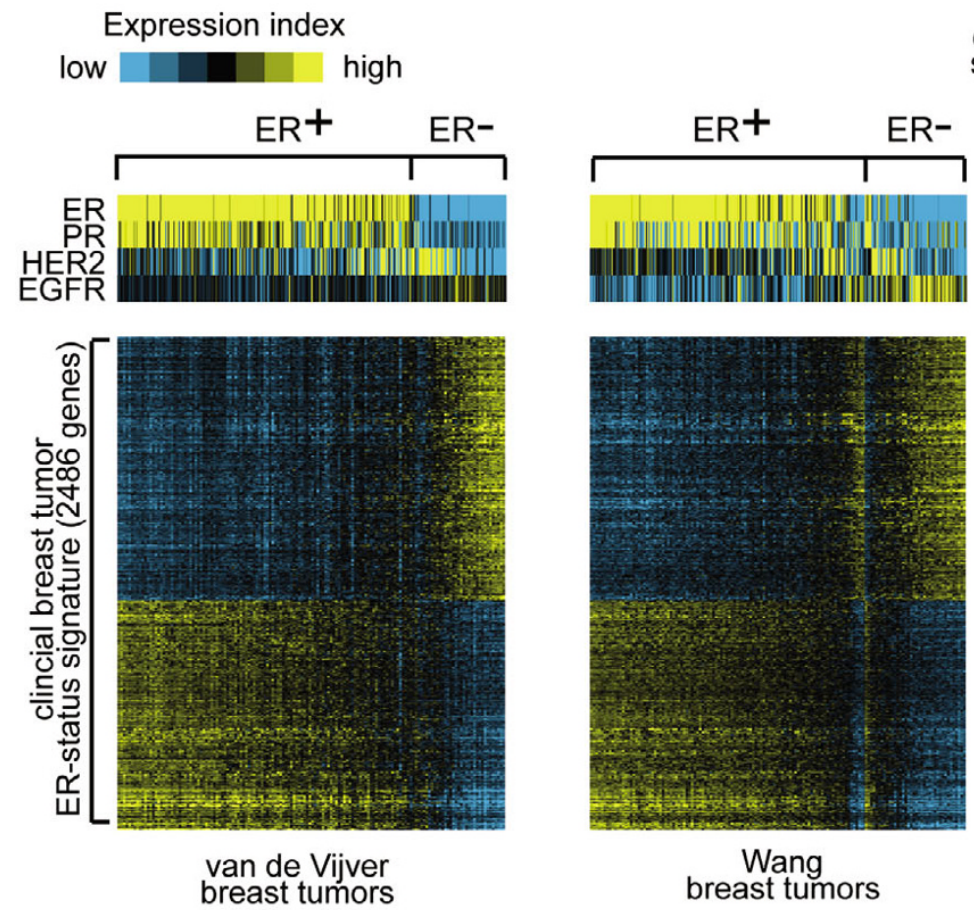

core breast ER-status
signature ( 417 genes)
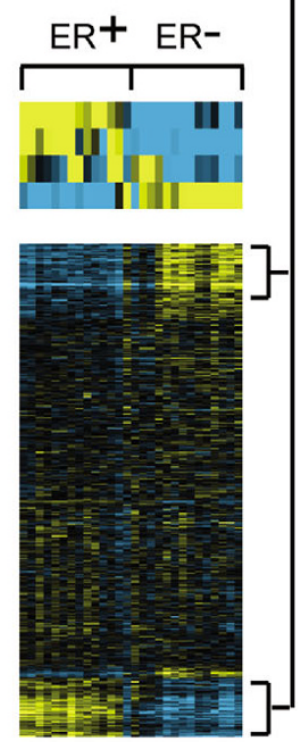

Bild

breast cell lines common signature of hormone independence (81 genes)
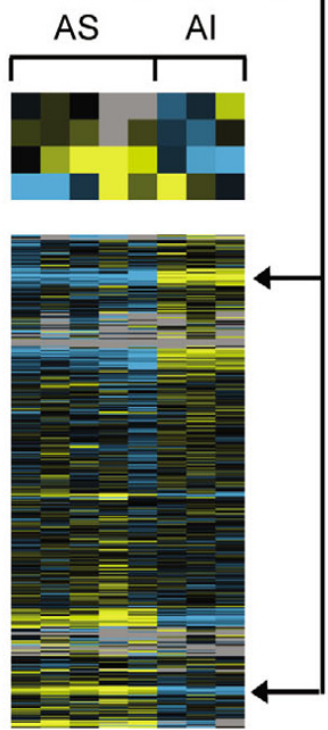

Zhao

prostate cell lines

\section{Figure 2}

Gene expression patterns of ER- clinical breast cancer are observed in both breast and prostate cancer cell lines. Heat map representation for 2486 unique named genes differentially expressed between ER+ and ER-breast tumors $(p<0.0 \mathrm{I}$ in both van de Vijver and Wang RNA profile datasets, I 332 higher in ER-). Expression patterns are represented as a color map. Each row represents a gene; each column represents a sample. The level of expression of each gene in each sample is represented using a yellow-blue color scale (yellow: high expression). Patterns corresponding to the 2486 genes are shown in both the Bild profile dataset of 18 breast cancer cell lines (ten ER-) and the Zhao dataset of eight prostate cell lines (AS, androgen sensitive; AI, androgen independent; gray denotes missing values or unrepresented genes). The order of the genes is the same for each of the datasets. Corresponding expression patterns for genes ER, PR, HER2, and EGFR are also shown. The order of the breast and prostate cell lines profiles is the same as that for Figure 4 (where they are labeled by name). Genes and associated expression values are available in Additional File I. 
Table I: Genes with elevated mRNA levels in common signature of hormone independence (Figure I)

\begin{tabular}{|c|c|c|c|c|c|}
\hline Entrez & Name & Title & Entrez & Name & Title \\
\hline 87 & ACTNI & actinin, alpha I & 5329 & PLAUR & plasminogen activator, urokinase receptor \\
\hline 136 & ADORA2B & adenosine $\mathrm{A} 2 \mathrm{~b}$ receptor & 5359 & PLSCRI & phospholipid scramblase I \\
\hline 390 & ARHE & Rho family GTPase 3 & 5621 & PRNP & Prion protein $(p 27-30)$ \\
\hline 824 & CAPN2 & calpain $2,(\mathrm{~m} / \mathrm{Il})$ large subunit & 6732 & SRPKI & SFRS protein kinase I \\
\hline 858 & CAV2 & caveolin 2 & 7272 & TTK & TTK protein kinase \\
\hline 994 & CDC25B & cell division cycle $25 \mathrm{~B}$ & 7296 & TXNRDI & thioredoxin reductase $\mathrm{I}$ \\
\hline 1075 & CTSC & cathepsin C & 7378 & UP & uridine phosphorylase I \\
\hline 1284 & COL4A2 & collagen, type IV, alpha 2 & 7398 & USPI & ubiquitin specific peptidase I \\
\hline 1786 & DNMTI & DNA (cytosine-5-)-methyltransferase I & 8882 & ZNF259 & zinc finger protein 259 \\
\hline 1969 & EPHA2 & $\mathrm{EPH}$ receptor $\mathrm{A} 2$ & 8898 & MTMR2 & myotubularin related protein 2 \\
\hline 2000 & ELF4 & E74-like factor 4 (ets domain) & 9056 & SLC7A7 & solute carrier family 7 , member 7 \\
\hline 2023 & ENOI & enolase I, (alpha) & 9322 & TRIPIO & thyroid hormone receptor interactor 10 \\
\hline 2037 & EPB4 IL2 & erythrocyte membrane protein band 4 .I-like 2 & 10403 & KNTC2 & kinetochore associated 2 \\
\hline 2131 & EXTI & exostoses (multiple) I & 10479 & SLC9A6 & solute carrier family 9 , member 6 \\
\hline 2182 & ACSL4 & acyl-CoA synthetase long-chain member 4 & 10644 & IMP-2 & IGF-II mRNA-binding protein 2 \\
\hline 2633 & GBPI & guanylate binding protein I, interferon-inducible & 10946 & SF3A3 & splicing factor $3 \mathrm{a}$, subunit $3,60 \mathrm{kDa}$ \\
\hline 2920 & CXCLI & chemokine (C-X-C motif) ligand 2 & 25937 & DKFZP5861I4I9 & WW domain containing transcription regulator I \\
\hline 3383 & ICAMI & $\begin{array}{l}\text { intercellular adhesion molecule I (CD54), human } \\
\text { rhinovirus receptor }\end{array}$ & 26031 & OSBPL3 & oxysterol binding protein-like 3 \\
\hline 3569 & IL6 & interleukin 6 (interferon, beta 2) & 26064 & RAII4 & retinoic acid induced 14 \\
\hline 3575 & IL7R & interleukin 7 receptor & 29083 & $\mathrm{HSPCI} 35$ & HSPCI 35 protein \\
\hline 3600 & ILI5 & interleukin 15 & 29970 & SCHIPI & schwannomin interacting protein I \\
\hline 3801 & KIFC3 & kinesin family member $\mathrm{C} 3$ & 29980 & DONSON & downstream neighbor of SON \\
\hline 3934 & LCN2 & lipocalin 2 (oncogene 24p3) & 55003 & PAKIIPI & PAKI interacting protein I \\
\hline 4478 & MSN & moesin & 56913 & CIGALTI & glycoprotein- $\mathrm{N}$-acetylgalactosamine 3-beta-galactosyltransferase \\
\hline 4907 & NT5E & 5'-nucleotidase, ecto (CD73) & 140885 & PTPNSI & protein tyrosine phosphatase, non-receptor type substrate I \\
\hline 5271 & SERPINB8 & serpin peptidase inhibitor, clade B & & & \\
\hline
\end{tabular}

From an mRNA profile data of eight different prostate cell lines, three of them AI, a set of 1793 genes that showed differential expression $(p<0.05)$ between AS and AI was obtained, 952 of these genes being higher in AI. The overlap of these 952 AI genes with the 223 core breast ER- signature (Figure 1) was 51; by chance, around 19 genes would have been expected to overlap, which makes the observed overlap of 51 highly significant ( $p=1 \mathrm{E}-11$, onesided Fisher's exact). A list of the 51 common prostate AI/ breast ER- genes is provided in Table 1 (heat map representation in Figure 2, associated gene expression values provided in Additional File 1). Only three genes in the list
(KNTC2, EXT1, and CDC25B) were annotated by Gene Ontology as having roles in the cell cycle or cell division, and so the 51 genes as a group do not appear to represent a program of general cellular proliferation.

The 952 prostate AI genes also shared highly significant overlap with the breast ER- clinical and breast ER-cell line gene sets individually ( 189 genes, Fisher's exact $p=1 \mathrm{E}-16$; and 194 genes, $p=1 \mathrm{E}-39$, respectively, see Figure 1). Similarly, the 841 genes higher in AS over AI prostate cell lines shared a highly significant overlap of 30 with the set of 194 in the core breast ER+ signature (expected 11, Fisher's

Table 2: Genes with diminished mRNA levels in common signature of hormone independence (Figure 2)

\begin{tabular}{|c|c|c|c|c|c|}
\hline Entrez & Name & Title & Entrez & Name & Title \\
\hline 367 & AR & androgen receptor & 7494 & XBPI & X-box binding protein I \\
\hline 388 & RHOB & ras homolog gene family, member B & 7644 & ZNF9I & zinc finger protein 91 \\
\hline 414 & ARSD & arylsulfatase D & 8349 & HIST2H2BE & Histone 2, $\mathrm{H} 2 \mathrm{be}$ \\
\hline 780 & DDRI & discoidin domain receptor family, member I & 10140 & TOBI & transducer of ERBB2, I \\
\hline 1153 & CIRBP & cold inducible RNA binding protein & 10229 & COQ7 & coenzyme Q7 homolog, ubiquinone \\
\hline 1154 & $\mathrm{CISH}$ & cytokine inducible SH2-containing protein & 11201 & POLI & polymerase (DNA directed) iota \\
\hline 1363 & CPE & carboxypeptidase $\mathrm{E}$ & 23199 & KIAA0I82 & KIAA0I 82 protein \\
\hline 2065 & ERBB3 & v-erb-b2 erythroblastic leukemia viral oncogene homolog 3 (avian) & 23247 & KIAA0556 & KIAA0556 protein \\
\hline 2746 & GLUDI & glutamate dehydrogenase I & 25800 & SLC39A6 & solute carrier family 39 , member 6 \\
\hline 2804 & GOLGBI & golgi autoantigen, golgin subfamily b, I & 27075 & TM4SFI3 & tetraspanin 13 \\
\hline 3169 & FOXAI & forkhead box Al & 27134 & TJP3 & tight junction protein 3 \\
\hline 3382 & $|C A|$ & islet cell autoantigen I, $69 \mathrm{kDa}$ & $5136 \mid$ & HOOKI & hook homolog I (Drosophila) \\
\hline 5241 & PGR & progesterone receptor & 51478 & HSDI7B7 & hydroxysteroid (I7-beta) dehydrogenase 7 \\
\hline 5349 & FXYD3 & FXYD domain containing ion transport regulator 3 & 55930 & MYO5C & Myosin VC \\
\hline 6509 & SLCIA4 & $\begin{array}{l}\text { solute carrier family I (glutamate/neutral amino acid transporter), member } \\
4\end{array}$ & 56654 & NPDCI & neural proliferation, differentiation and control, I \\
\hline
\end{tabular}




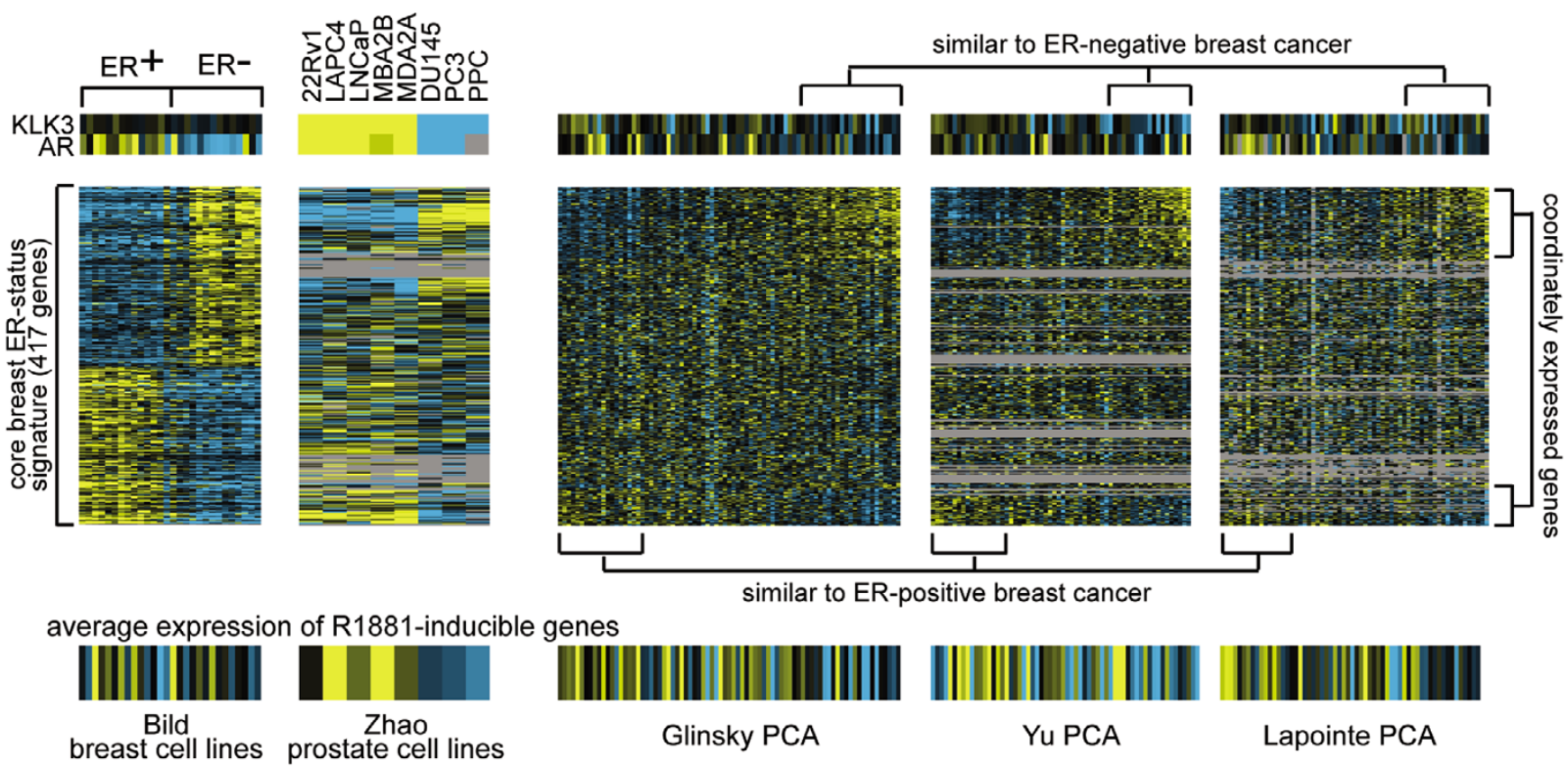

\section{Figure 3}

Gene expression patterns of ER- breast cancer are observed in a subset of clinically localized prostate cancer (PCA). Heat map representation for 4I7 unique genes differentially expressed between ER+ and ER- clinical breast tumors and cell lines (from Figure 2). The patterns corresponding to these genes are shown in three independent RNA profile datasets of PCA from Glinsky et al. [14], Yu et al. [15], and Lapointe et al. [16]. The PCA profiles are ordered from those that share more similarity with the ER+ breast cancer pattern to those that share more similarity with the ER- breast cancer pattern. The order of the genes is the same for each dataset represented. For each dataset, the average expression of a set of 559 genes induced by synthetic androgen RI88I in vitro in a dataset from Chen et al. [18] is also represented. The order of the breast and prostate cell lines profiles is the same as that for Figure 4. Genes and associated expression values are available in Additional File 2.

exact $p=1 \mathrm{E}-07)$. A list of these 30 common prostate AS/ breast ER+ genes is provided in Table 2. Of the 30 genes, six (CISH, KIAA0182, ICA1, PGR, SLC1A4, XBP1) were upregulated by estrogen signaling (in cluster " $B$ " from ref 13) and six (KIAA0182, GLUD1, FOXA1, FXYD3, NPDC1, HIST2H2BE) were up-regulated $(p<0.001)$ by androgen signaling, based on analysis of data from published RNA profiling studies of breast cancer [13] and prostate cancer [14] cell cultures, respectively.

Enrichment of the prostate AI/AS gene signature within the breast cell line ER-/ER+ signature was also demonstrated using an alternative analytical technique (known as "Q1-Q2" in Tian et al., ref 15), in which all the genes represented in the Bild breast cell line dataset were ranked by over-expression in ER- over ER+ cell lines, and the relative positions of the prostate AI and AS gene sets were each evaluated within the ranked list from the breast dataset. Over any randomly selected set of genes from the prostate dataset, and over any random assignment of the profile labels in the breast dataset, the AI genes were enriched within the ER- genes and the AS genes, within the ER+ genes ( $p=0.0001$ and $p=0.01$, respectively). The 81 genes concurrent between $\mathrm{AI} / \mathrm{AS}$ prostate cancer and the ER-/ER+ prostate signatures was termed a common signature of hormone independence. While these 81 genes represented significant similarities between hormone independent breast and prostate cancers, there were many more genes not shared between the two (Figures 1 and 2), as would expected when comparing these two rather different systems.

\section{Identification of a subset of clinically localized prostate tumors having the gene signature of hormone independence and showing repression of androgen signaling}

Primary IBC presents as either hormone dependent (ER+) or independent (ER-). Based on the observed overlap between an in vitro gene signature of androgen independence in prostate cancer and the signature of estrogen independence in clinical breast tumors (Figures 1 and 2), it seemed plausible that a subset of androgen independent primary prostate tumors could be defined using the breast tumor expression profile data. Three independent mRNA profile datasets of clinically localized prostate cancer (PCA) were considered: from Glinsky et al. [16] of 79 tumors, from Yu et al. [17] of 60 tumors, and from Lapointe et al. [18] of 62 tumors. The PCA profiles in each 
A
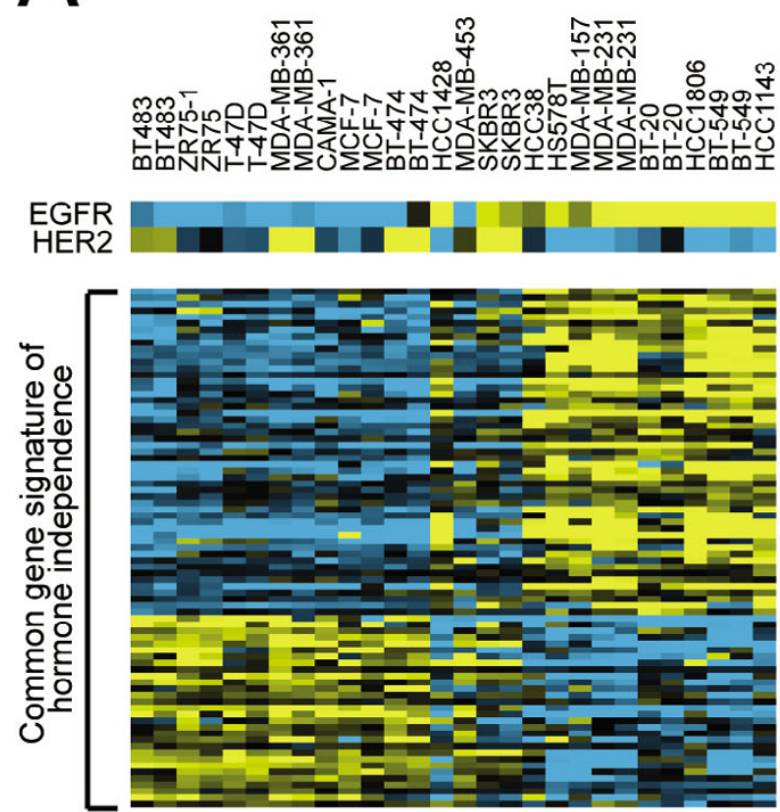

B
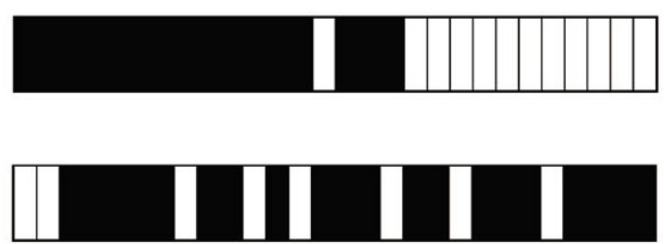

Prostate cancer cell lines
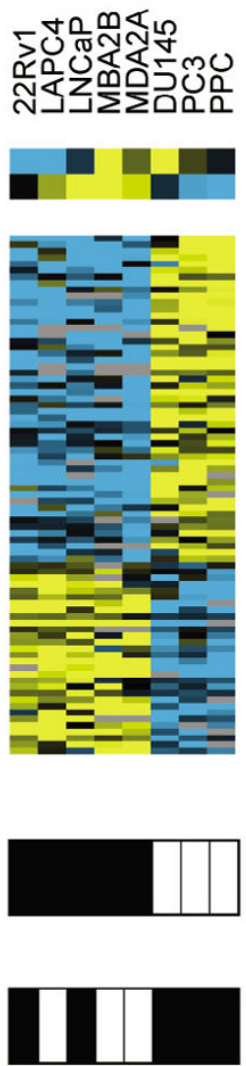

white - widespread similarities to MCF7 EGFR gene signature oncogene-activated
MCF7 cultures
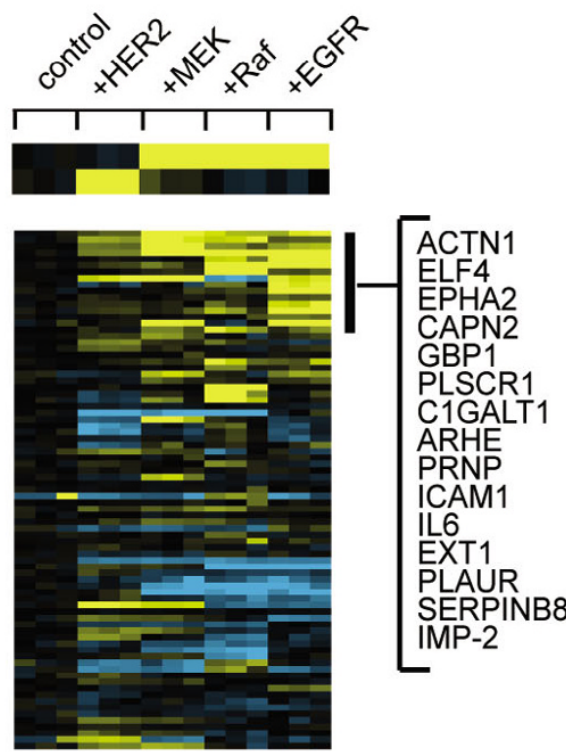

white - widespread similarities to MCF7 HER2 gene signature

\section{Figure 4}

Genes associated with hormone independence in both breast and prostate cancer are enriched for transcriptional targets of the EGFR signaling pathway. (A) Heat map representation for $8 \mathrm{I}$ unique genes in the common signature of hormone independence (from Figures I and 2). The patterns corresponding to these genes are shown in the breast and prostate cell line profile datasets and in a dataset from Creighton et al. [2I] of MCF-7 cell lines with activated oncogenes HER2, MEK, Raf, or EGFR. The order of the genes is the same for the datasets. The set of genes both associated with ER- and AS cell lines and activated by EGFR $(p<0.0 \mathrm{I})$ are highlighted. (B) Classification of the breast and prostate cell lines as "EGFR-like" or "ERBB2-like," using the entire sets of genes from the Creighton dataset that were differentially expressed $(p<0.01$, irrespective of the Bild and Zhao datasets) in the EGFR or HER2 MCF-7 cell lines, respectively, as compared to controls. Genes and associated expression values are available in Additional File 3.

of the three datasets were ordered based on the extent of similarity in expression patterns (by Pearson's correlation coefficient) with that of the 417 genes in the core breast ER-status signature (Figure 2).

For each PCA profile dataset, a sizable fraction of the tumors were significantly correlated $(p<0.01)$ with the ERstatus signature (Glinsky: 52\%, Yu: 52\%, Lapointe: 32\%), some of the PCA having patterns similar to ER- IBC, others to ER+ IBC. When selecting a random set of 417 genes from the Glinsky dataset to represent the ER-status signature, none of Glinsky tumors shared significant similarities to the random pattern as expected. These observations indicated that the set of genes associated with hormone independence in breast tumors are coordinately expressed in PCA, which was further evident when viewing the associated expression patterns as heat maps (Figure 3, associated gene expression values provided in Additional File 2). For the genes high in ER- IBC, a sizable fraction were also high in a subset of the PCA tumors; in these same tumors, a sizable fraction of the ER+ genes were down. Where the breast cancer cell lines and the PCA shared common expression patterns, the associated genes also showed concordant expression in the prostate cell line data. When comparing the subset of clinical PCA having significant similarities $(p<0.01)$ to ER- breast tumors and cell lines with the subset of PCA similar to ER+ breast, the androgen receptor (AR) was significantly decreased 
$(p<0.01)$ in PCA similar to ER- breast for the Glinsky and Lapointe datasets but not the Yu datasets. KLK3, which encodes prostate-specific antigen (PSA), was decreased $(p<0.01)$ in PCA similar to ER- breast for the Yu and Glinksy but not the Lapointe datasets.

Transcriptional targets of the androgen signaling pathway have been defined previously using gene expression profiling of cell cultures [14,19]. From the profile dataset from Chen et al. [14], a set of 559 unique named genes showing induction $(p<0.001)$ by synthetic androgen R1881 were obtained. Relatively few genes in the common signature of hormone independence (Figure 2) were androgen-inducible, six of them in the set of 30 AS/ER+ genes and three in the set of $51 \mathrm{AI} / \mathrm{ER}$ - genes. Of the 223 ER- genes and $194 \mathrm{ER}+$ genes in the core ER-status signature, 22 and 13, respectively, were R1881-inducible. When comparing the subset of clinical PCA having significant similarities $(p<0.01)$ to ER- breast with the subset of PCA similar to ER+ breast, the PCA similar to ER- showed lower average expression of the R1881-inducible genes (Glinsky $p<0.0002, \mathrm{Yu} p<0.0007$, Lapointe $p<0.09$, $t$-test, see also Figure 3). Across all of the PCA profiles, the $t$-statistic of the similarity with the ER-core signature pattern was inversely correlated ( $p<0.05$, Pearson's) with the average expression of R1881-inducible genes in each of the three datasets. These patterns indicated that the androgen signaling pathway tends to be deactivated or suppressed in PCA exhibiting the gene signature of hormone independence.

\section{ER- breast and Al prostate cell lines are significantly enriched for transcriptional targets of the EGFR pathway} For clues as to what molecular pathways may be represented in the common gene signature of hormone independence (Figure 2), transcriptional targets of various pathways from public datasets were examined. Pathways considered included: Myc, c-Src, beta-catenin, E2F3, and $\mathrm{H}$-Ras, from the expression profile dataset from Bild et al. [11]; Akt, from the dataset by Majumder et al. [20]; cyclin D1, from the dataset by Lamb et al. [21]; and Her2, EGFR, MEK, and Raf, from the dataset by Creighton et al. [22]. In the previous Creighton study, ER+ MCF-7 breast cancer cells were made to stably over-express EGFR or constitutively activate erbB-2, Raf, or MEK; which resulted in these cells exhibiting estrogen-independent growth and the down-regulation of ER expression. Of all the pathway gene signatures considered in this present study, the EGFR, MEK, and Raf signatures shared significant similarities with the common hormone independence signature. Of the 734 genes up-regulated $(p<0.01)$ by EGFR, the 1238 genes up-regulated by MEK, and the 618 up-regulated by Raf: 16, 15, and 12, respectively, were shared with the 51 AI prostate/ER- breast genes of Table 1 (one-sided Fisher's exact $p<4 \mathrm{E}-09, p<2 \mathrm{E}-05$, and $p<2 \mathrm{E}-06$, respec- tively). Conversely, of the 940 genes down-regulated by EGFR, the 1182 genes down-regulated by MEK, and the 988 down-regulated by Raf: 11 , 8, and 15, respectively, were shared with the $30 \mathrm{AS}$ prostate/ER+ breast genes of Table 2 (Fisher's exact $p<2 \mathrm{E}-06, p=0.003$, and $p<3 \mathrm{E}-10$, respectively).

The expression patterns of the hormone independence signature were viewed as a heat map in the context of the patterns of the MCF7 cell lines with activated HER2, MEK, Raf, or EGFR (Figure 4A, associated gene expression values provided in Additional File 3). Most of the ER- breast tumors and cell lines over-expressed EGFR mRNA, and those that did not tended to over-expressed HER2 (Figure 2, Figure 4A). The AI prostate cell lines, however, did show over-expression of HER2 at the mRNA level. In addition, using the entire set of 1674 unique genes differentially expressed in the MCF7-EGFR cell line relative to control ( $p<0.01,734$ up-regulated), the Bild breast and Zhao prostate cell lines were stratified based on similarity ( $p<0.01$, Pearson's correlation) to the EGFR gene signature pattern (Figure 4B). Most all of the ER- breast cell lines (with the exception of MDA-MD-453 and HER2+ SKBR3), all of AI prostate cell lines, and none of the ER+ or AS cell lines (with the exception of HCC1428) shared extensive similarities with the EGFR transcriptional signature. A similar analysis was carried out using the HER2 gene signature, but no stratification on the basis of hormone insensitivity was observed. The hormone independence signature showed no enrichment for HER2 transcriptional targets (Figure 4A).

\section{Discussion}

One of the "holy grails" of both breast and prostate cancer research is to determine how these cancers acquire hormone independence and how best to treat them when they do. A number of molecular mechanisms have been postulated to explain resistance to hormone therapy in prostate cancer. One class of resistant prostate tumors continues to rely upon androgen receptor (AR) signaling through a number of means, including: over-expression of the AR gene, through DNA amplification or some other mechanism [14,23]; "promiscuous" point mutation in $A R$, allowing the receptor to be activated by steroids other than androgen, including anti-androgens and estrogen [24]; and ligand-independent activation of AR, mediated by oncogenes such as ERBB2 or HRAS [25]. While tumors that fall under the above may often be referred to as "androgen independent" [4], for the purposes of this present study we must draw a distinction between the above class of hormone therapy resistant prostate cancer and a second class, which bypasses AR function completely and does not rely upon androgens for growth. This second class is what appears to be involved in the gene signature of hormone independence uncovered here as 
being common to both ER- breast cancer and prostate cancer that is completely AI.

The specific alternative signaling pathways that allow prostate tumors to bypass AR have been somewhat elusive, one candidate pathway possibly involving BCL2 [4]. This present study has implicated EGFR signaling has playing an important role in bypassing AR. While transcriptional targets of EGFR were enriched in AI prostate cancer (Figure 4), EGFR mRNA itself did not appear elevated in AI cell lines. Neither was EGFR mRNA consistently elevated in the clinical PCA samples that exhibited an ER-/AI molecular phenotype (Figure 3). However, EGFR protein itself is elevated in AI over AS cell lines [26]. Recent studies have found EGFR protein expression in tumor tissues to be strongly associated with hormone refractory status [27-29]. The EGFR tyrosine kinase inhibitor gefitinib causes cell cycle arrest and initiates apoptosis in primary PCA cultures and in PCA cell lines, including DU145 and PC3 as well as LNCaP [26]. In studies using the AI cell line PC3, suppression of EGF-R signaling reduced the incidence of prostate cancer metastasis in nude mice [30].

This present study indicates that a subset of primary PCA presents as hormone independent, prior to patient treatment with adjuvant anti-androgen therapy (Figure 3). It has been previously thought that a sub-population of androgen-resistant cells might coexist with androgendependent cells within the tumor, and that anti-androgen therapy would therefore kill off the dependent cells and leave the resistant cells to thrive [1]. This study lends support to an adjuvant therapy strategy of combining EGFR inhibitors with anti-androgens. One question to consider in designing clinical trials testing this treatment regimen is whether PSA recurrence would be a suitable endpoint, as AI prostate cancer may not express PSA (Figure 3). The ability to identify a hormone independent subset of primary breast cancer using ER as a biomarker has implications for selecting the course of adjuvant treatment [3]. If a subset of primary prostate tumors could be diagnosed in the clinic as being hormone independent, it could warrant more aggressive treatment with alternative therapies to anti-androgens. Individual genes in the list of 81 in the gene signature of hormone independence might be good candidates for prognostic markers in PCA, or several genes in the signature could perhaps be used together.

In other studies, gene expression profiling has been carried out on hormone refractory metastases of prostate cancer [31,32]. One issue with comparing results derived from those datasets with this present study's gene signature of hormone independence is that, as discussed above, these metastases likely represent several mechanisms of hormone therapy resistance, not simply the use of EGFRmediated bypass of AR function, as appears to be mani- fested in the AI prostate cell lines analyzed here. Genes expressed in the EGFR-dependent AI subtype may not be uniformly expressed in all varieties of hormone refractory cancers. The dataset from ref [32] was analyzed here in the context of the datasets used in this study; when considering the genes high in hormone-refractory prostate metastases compared to PCA, no significant overlap of these genes was observed with the genes high in the AI prostate cell lines or the ER- breast tumors (results not shown). A number of expression profiling studies using prostate tumor xenografts acquiring resistance to hormone therapy have been carried out [14,33-36]; many of these studies appear to represent cancers that develop hypersensitivity to androgen pathway activation, rather than androgen pathway independence. The hormone independent gene signature of this present study did not show coordinate expression in a profile dataset from Chen et al. [14] of hormone therapy-resistant prostate tumors xenografts (results not shown); as these tumor xenografts uniformly up-regulated $A R$, it could be presumed that these xenografts represented a model of increased sensitivity to androgen levels.

One limitation with this present study is the small number of prostate cancer cell lines for which gene expression profile data was available (five AS and three AI). Profiling studies in breast cancer indicate that there are at least two subtypes of ER- IBC, a HER2+ subtype and a "basal" subtype $[6,7,12]$. Interestingly, recent studies indicate that a subset of ER- breast cancer may rely upon the androgen pathway rather than the estrogen pathway $[37,38]$, though this does not appear to represent the subset of ER-considered here, as androgen-regulated genes were not enriched in the ER- gene signature of Figure 2 (results not shown). If more prostate cancer cell lines were profiled, it might uncover additional subtypes of AI PCA to the EGFR-dependent subtype uncovered here. The basal subtype of ER- IBC also appears to rely upon EGFR signaling (Figure 4). A number of clinical trials testing the efficacy of EGFR tyrosine kinase inhibitors (TKIs) in breast cancer treatment are underway. Initial phase II studies have suggested that the EGFR TKIs do not have a high efficacy in a heavily pre-treated population of patients with metastatic breast cancer; however, in patients with hormone therapy-resistant ER+ tumors, EGFR inhibition does appear to have a significant therapeutic effect [39]. In at least one small trial, there was evidence of only minimal efficacy in advanced, metastatic ER- breast cancer [39], though one could make the conjecture that antiEGFR therapy might be more effective in less advanced cancers.

The 81 genes in the signature of hormone independence common to prostate and breast is a much shorter set compared to the hundreds of genes associated separately with either prostate or breast (Figures 1 and 2). One may 
expand the set of 81 somewhat by using slightly less stringent statistical cutoffs. At the same time, the set of 81 may provide a good starting point for further study. Interestingly, a number of the 51 genes higher in AI prostate and ER- breast were previously associated with the immune response, including ELF4, GBP1, CXCL2, ILG, IL7R, and IL15. IL6 (interleukin 6) in particular - indicated here to be a transcriptional target of the EGFR pathway (Figure 4) - has been shown to promote prostate tumor growth and to play a role in the interaction between epithelial and stromal cells in prostate cancer [40]. One next step in studying these genes would likely be validation of their expression patterns in breast and prostate tissues or cell lines, using some alternative technique from microarrays, such as westerns or quantitative RT-PCR; it is expected, however, that most of the genes in the set of 81 would validate, as their expression patterns were observed in multiple profile datasets generated on different microarray platforms, which in itself could be considered validation [41].

\section{Conclusion}

In conclusion, the hope for this study is that it may aid in the development of therapy regimens to target the subset of breast and prostate cancers that up until the present have been the most difficult to treat.

\section{Methods}

The gene expression profile datasets used in this study were all publicly available. From the 66 PCA profiles described in the study by Yu et al. [15], 60 were available for this present study (collection for this dataset was facilitated by A.M. Chinnaiyan and the Oncomine team). Recent evidence emerged that the MDA-MB-435 cell line was not breast but melanoma [42] and so the MDA-MD435 profiles in the Bild dataset [11] were removed from the analysis. Gene expression values in each dataset were log-transformed. Gene expression values in the clinical breast tumor and cell line profile datasets were centered on the centroid mean of ER- and ER+. Values in the Zhao prostate cell line profile dataset [8] were centered on the centroid mean of AS and AI. Clinical prostate tumor datasets were transformed to standard deviations from the median. For the androgen dataset from Chen et al. [14], expression values within the AR+ group of samples were transformed to standard deviations from the mean; values within the vector group of samples were separately transformed. Expression values in the Creighton MCF-7 dataset [22] were centered on the mean of the MCF-7/lt-E2 control group.

As the expression profile datasets were generated on different platforms, and as many of the genes represented were measured on multiple probes in any one dataset, a method to select the optimal probe to represent each gene in an unbiased fashion was used when joining multiple datasets. For the Affymetrix array datasets, the probe with the greatest variation across samples represented the gene. For the cDNA microarray datasets (Zhao cell line and Lapointe PCA), the probe with the most unflagged values across samples, followed by the probe with the greatest variation, represented the genes. The Entrez Gene identifier was used in mapping genes across datasets. Two-sample $t$-tests determined significant differences in gene expression between groups of samples. For the Chen androgen dataset, the Pearson's correlation between gene expression and the log of the concentration of R1881 determined significance of R1881 induction. Expression values were visualized as heat maps using the Cluster [43] and Java TreeView software [44]. Prior to heat map generation, genes were manually sorted using Microsoft Excel in order to highlight gene groups of interest.

The one-sided Fisher's exact test determined significance of overlap between any two distinct sets of genes. Q1-Q2 enrichment analysis [15] was carried out essentially as described in ref [45]. For "stratifying" a set of profiles in a given dataset on the basis of a pre-defined expression pattern (e.g. stratifying the clinical PCA profiles using the ERstatus gene signature in Figure 3), each gene involved in the pattern was represented as " 1 " or "-1" (for up or down, respectively), and the Pearson's correlation coefficient was computed between the pattern and each individual profile (with significance by two-sided $t$-test).

\section{Abbreviations}

estrogen receptor alpha (ER), progesterone receptor (PR), invasive breast cancer (IBC), clinically localized prostate cancer (PCA), androgen independent (AI), androgen sensitive (AS)

\section{Authors' contributions}

CC conceived of the study, collected the publicly available datasets, did the analysis, and wrote the manuscript.

\section{Additional material}

\section{Additional File 1}

Supporting Data, Figure 2. Excel worksheets with the expression data that was presented as heat maps in Figure 2, along with a list of the associated genes.

Click here for file

[http://www.biomedcentral.com/content/supplementary/1471-

2164-8-199-S1.zip] 


\section{Additional File 2}

Supporting Data, Figure 3. Excel worksheets with the expression data that was presented as heat maps in Figure 3, along with a list of the associated genes.

Click here for file

[http://www.biomedcentral.com/content/supplementary/1471-

2164-8-199-S2.xls]

\section{Additional File 3}

Supporting Data, Figure 4. Excel worksheets with the expression data that was presented as heat maps in Figure 4, along with a list of the associated genes.

Click here for file

[http://www.biomedcentral.com/content/supplementary/14712164-8-199-S3.xls]

\section{Acknowledgements}

The author would like to thank all contributors of the published datasets that were used in this study.

\section{References}

I. Abate-Shen C, Shen MM: Molecular genetics of prostate cancer. Genes Dev 2000, I 4:24I 0-34.

2. Elledge RM, Fuqua SA: Estrogen and progesterone receptors. In Diseases of the Breast Edited by: Harris J, Lippman ME, Morrow M, Osborne C. Philadelphia: Lippincott, Williams and Wilkins; 2000:47I-488.

3. Allred DC, Brown P, Medina D: The origins of estrogen receptor alpha-positive and estrogen receptor alpha-negative human breast cancer. Breast Cancer Res 2004, 6:240-5.

4. Feldman BJ, Feldman D: The development of androgen-independent prostate cancer. Nat Rev Cancer 200I, I:34-45.

5. Clark GM, McGuire WL: Steroid receptors and other prognostic factors in primary breast cancer. Semin Oncol 1988, 15:20-25.

6. Sorlie T, Perou CM, Tibshirani R, Aas T, Geisler S, Johnsen H, Hastie $T$, Eisen MB, van de Rijn M, Jeffrey SS, et al.: Gene expression patterns of breast carcinomas distinguish tumor subclasses with clinical implications. Proc Natl Acad Sci USA 200 I, 98: I 0869- 10874

7. Sorlie T, Tibshirani R, Parker J, Hastie T, Marron JS, Nobel A, Deng S, Johnsen H, Pesich R, Geisler S, et al:: Repeated observation of breast tumor subtypes in independent gene expression data sets. Proc Natl Acad Sci USA 2003, I 00:84 I8-23.

8. Zhao H, Kim Y, Wang P, Lapointe J, Tibshirani R, Pollack JR, Brooks JD: Genome-wide characterization of gene expression variations and DNA copy number changes in prostate cancer cell lines. Prostate 2005, 63: $187-197$.

9. van de Vijver MJ, He YD, van't Veer LJ, Dai H, Hart AA, Voskuil DW, Schreiber GJ, Peterse JL, Roberts C, Marton MJ, et al.: A geneexpression signature as a predictor of survival in breast cancer. N Engl J Med 2002, 347:1999-2009.

10. Wang Y, Klijn JG, Zhang Y, Sieuwerts AM, Look MP, Yang F, Talantov D, Timmermans M, Meijer-van Gelder ME, Yu J, et al.: Gene-expression profiles to predict distant metastasis of lymph-nodenegative primary breast cancer. Lancet 2005, 365:67|-9.

II. Bild AH, Yao G, Chang JT, Wang Q, Potti A, Chasse D, Joshi MB, Harpole D, Lancaster JM, Berchuck A, et al:: Oncogenic pathway signatures in human cancers as a guide to targeted therapies. Nature 2006, 439:353-7.

12. Neve RM, Chin K, Fridlyand J, Yeh J, Baehner FL, Fevr T, Clark L, Bayani N, Coppe JP, Tong F, et al:: A collection of breast cancer cell lines for the study of functionally distinct cancer subtypes. Cancer Cell 2006, 10:5 15-27.

13. Creighton CJ, Cordero KE, Larios JM, Miller RS, Johnson MD, Chinnaiyan AM, Marc E, Lippman ME, Rae JM: Genes regulated by estrogen in breast tumor cells in vitro are similarly regulated in vivo in tumor xenografts and human breast tumors. Genome Biol 2006, 7:R28.
14. Chen CD, Welsbie DS, Tran C, Baek SH, Chen R, Vessella R, Rosenfeld MG, Sawyers CL: Molecular determinants of resistance to antiandrogen therapy. Nat Med 2004, 10:33-9.

15. Tian L, Greenberg SA, Kong SW, Altschuler J, Kohane IS, Park PJ: Discovering statistically significant pathways in expression profiling studies. Proc Natl Acad Sci USA 2005, 102: $13544-9$.

16. Glinsky GV, Glinskii AB, Stephenson AJ, Hoffman RM, Gerald WL: Gene expression profiling predicts clinical outcome of prostate cancer. J Clin Invest 2004, I I 3:9 | 3-23.

17. Yu YP, Landsittel D, Jing L, Nelson J, Ren B, Liu L, McDonald C, Thomas R, Dhir R, Finkelstein S, et al.: Gene expression alterations in prostate cancer predicting tumor aggression and preceding development of malignancy. J Clin Oncol 2004, 22:2790-9.

18. Lapointe J, Li C, Higgins JP, van de Rijn M, Bair E, Montgomery K, Ferrari M, Egevad L, Rayford W, Bergerheim U, et al.: Gene expression profiling identifies clinically relevant subtypes of prostate cancer. Proc Natl Acad Sci USA 2004, I $01: 8$ I I -6.

19. DePrimo SE, Diehn M, Nelson JB, Reiter RE, Matese J, Fero M, Tibshirani R, Brown PO, Brooks JD: Transcriptional programs activated by exposure of human prostate cancer cells to androgen. Genome Biol 2002, 3:R32.

20. Majumder PK, Febbo PG, Bikoff R, Berger R, Xue Q, McMahon LM, Manola J, Brugarolas J, McDonnell T], Golub TR, et al: $\mathbf{m T O R}$ inhibition reverses Akt-dependent prostate intraepithelial neoplasia through regulation of apoptotic and HIF-I-dependent pathways. Nat Med 2004, 10:594-60I.

21. Lamb J, Ramaswamy S, Ford HL, Contreras B, Martinez RV, Kittrell FS, Zahnow CA, Patterson N, Golub TR, Ewen ME: A mechanism of cyclin DI action encoded in the patterns of gene expression in human cancer. Cell 2003, I I 4:323-34.

22. Creighton CJ, Hilger AM, Murthy S, Rae JM, Chinnaiyan AM, El-Ashry D: Activation of mitogen-activated protein kinase in estrogen receptor alpha-positive breast cancer cells in vitro induces an in vivo molecular phenotype of estrogen receptor alpha-negative human breast tumors. Cancer Res 2006, 66:3903-II.

23. Visakorpi T, Hyytinen E, Koivisto P, Tanner M, Keinanen R, Palmberg C, Palotie A, Tammela T, Isola J, Kallioniemi OP: In vivo amplification of the androgen receptor gene and progression of human prostate cancer. Nat Genet 1995, 9:40I-406.

24. Veldscholte J, Berrevoets CA, Ris-Stalpers C, Kuiper GG, Jenster G, Trapman J, Brinkmann AO, Mulder E: The androgen receptor in LNCaP cells contains a mutation in the ligand binding domain which affects steroid binding characteristics and response to antiandrogens. J Steroid Biochem Mol Biol 1992, 41:665-669.

25. Craft N, Shostak Y, Carey M, Sawyers CL: A mechanism for hormone-independent prostate cancer through modulation of androgen receptor signaling by the HER-2/neu tyrosine kinase. Nat Med 1999, 5:280-285

26. Vicentini C, Festuccia C, Gravina GL, Angelucci A, Marronaro A, Bologna $M$, et al.: Prostate cancer cell proliferation is strongly reduced by the epidermal growth factor receptor tyrosine kinase inhibitor ZD 1839 in vitro on human cell lines and primary cultures. J Cancer Res Clin Oncol 2003, I 29:165-174

27. Di Lorenzo G, Tortora G, D'Armiento FP, De Rosa G, Staibano S, Autorino R, D'Armiento M, De Laurentiis M, De Placido S, Catalano $\mathrm{G}$, et al.: Expression of epidermal growth factor receptor correlates with disease relapse and progression to androgenindependence in human prostate cancer. Clin Cancer Res 2002, 8:3438-3444.

28. Bartlett JM, Brawley D, Grigor K, Munro AF, Dunne B, Edwards J: Type I receptor tyrosine kinases are associated with hormone escape in prostate cancer. I Pathol 2005, 205:522-529.

29. Shah RB, Ghosh D, Elder JT: Epidermal growth factor receptor (ErbBI) expression in prostate cancer progression: correlation with androgen independence. Prostate 2006, 66: I437-I 444

30. Angelucci A, Gravina GL, Rucci N, Millimaggi D, Festuccia C, Muzi P, Teti A, Vicentini C, Bologna M: Suppression of EGF-R signaling reduces the incidence of prostate cancer metastasis in nude mice. Endocr Relat Cancer 2006, 13:197-210.

31. Stanbrough M, Bubley G], Ross K, Golub TR, Rubin MA, Penning TM, Febbo PG, Balk SP: Increased expression of genes converting adrenal androgens to testosterone in androgen-independent prostate cancer. Cancer Res 2006, 66:28I5-25. 
32. Tomlins SA, Mehra R, Rhodes DR, Cao X, Wang L, Dhanasekaran SM, Kalyana-Sundaram S, Wei JT, Rubin MA, Pienta KJ, Shah RB, Chinnaiyan AM: Integrative molecular concept modeling of prostate cancer progression. Nat Genet 2007, 39:4I-5I.

33. Bubendorf L, Kolmer M, Kononen J, Koivisto P, Mousses S, Chen $Y$, Mahlamäki E, Schraml P, Moch H, Willi N, Elkahloun AG, Pretlow TG, Gasser TC, Mihatsch MJ, Sauter G, Kallioniemi OP: Hormone therapy failure is human prostate cancer: analysis by complementary DNA and tissue microarrays. J Natl Cancer Inst 1999, 91:1758-1764.

34. Mousses S, Wagner U, Chen Y, Kim JW, Bubendorf L, Bittner M, Pretlow T, Elkahloun AG, Trepel JB, Kallioniemi OP: Failure of hormone therapy in prostate cancer involves systematic restoration of androgen responsive genes and activation of rapamycin sensitive signaling. Oncogene 200I, 20:67I8-6723.

35. Amler LC, Agus DB, LeDuc C, Sapinoso ML, Fox WD, Kern S, Lee D, Wang V, Leysens M, Higgins B, Martin J, Gerald W, Dracopoli N, Cordon-Cardo C, Scher HI, Hampton GM: Dysregulated expression of androgen-responsive and nonresponsive genes in the androgen-independent prostate cancer xenograft model CWR22-R. Cancer Res 2000, 60:6I34-6I4I.

36. Sirotnak FM, She Y, Khokhar NZ, Hayes P, Gerald W, Scher HI: Microarray analysis of prostate cancer progression to reduced androgen dependence : studies in unique models contrasts early and late molecular events. Mol Carcinog 2004, $41: 150-163$.

37. Doane AS, Danso M, Lal P, Donaton M, Zhang L, Hudis C, Gerald WL: An estrogen receptor-negative breast cancer subset characterized by a hormonally regulated transcriptional program and response to androgen. Oncogene 2006, 25:3994-4008.

38. Farmer $\mathrm{P}$, Bonnefoi $\mathrm{H}$, Becette $\mathrm{V}$, Tubiana-Hulin $\mathrm{M}$, Fumoleau $\mathrm{P}$, Larsimont D, Macgrogan G, Bergh J, Cameron D, Goldstein D, Duss S, Nicoulaz AL, Brisken C, Fiche M, Delorenzi M, Iggo R: Identification of molecular apocrine breast tumours by microarray analysis. Oncogene 2005, 24:4660-7I.

39. Agrawal A, Gutteridge E, Gee JM, Nicholson RI, Robertson JF: Overview of tyrosine kinase inhibitors in clinical breast cancer. Endocr Relat Cancer 2005, I 2(SuppI I):SI 35-44.

40. Hughes C, Murphy A, Martin C, Sheils O, O'Leary J: Molecular pathology of prostate cancer. J Clin Pathol 2005, 58:673-84.

4I. Rhodes DR, Barrette TR, Rubin MA, Ghosh D, Chinnaiyan AM: Meta-analysis of microarrays: interstudy validation of gene expression profiles reveals pathway dysregulation in prostate cancer. Cancer Res 2002, 62:4427-4433.

42. Rae JM, Creighton CJ, Meck JM, Haddad BR, Johnson MD: MDA-MB435 cells are derived from MI4 Melanoma cells - a loss for breast cancer, but a boon for melanoma research. Breast Cancer Res Treat 104(I): 13-19. Epub Sep 272006

43. Eisen MB, Spellman PT, Brown PO, Botstein D: Cluster analysis and display of genome-wide expression patterns. Proc Natl Acad Sci USA 1998, 95: 14863-14868.

44. Saldanha AJ: Java Treeview - extensible visualization of microarray data. Bioinformatics 2004, 20:3246-8.

45. Creighton CJ: A gene transcription signature of the Akt/ mTOR pathway in clinical breast tumors. Oncogene. E-pub Jan 82007

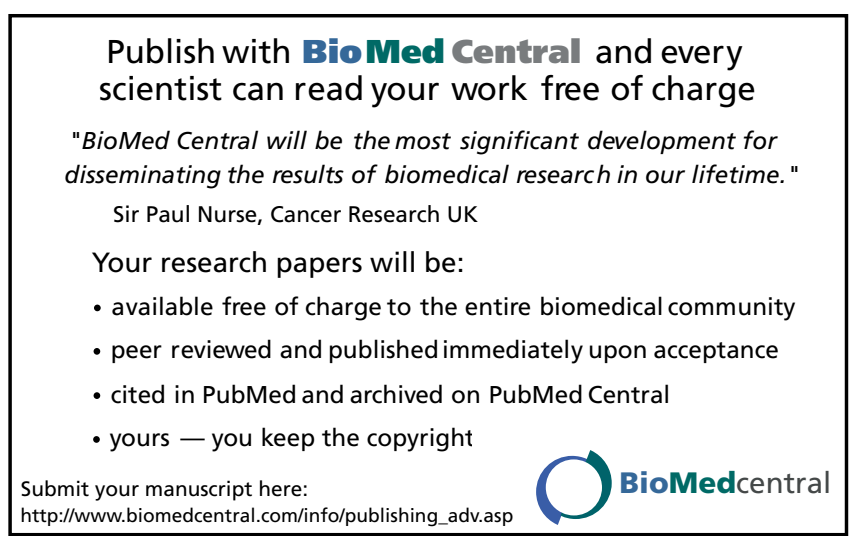

